\title{
Abschied und Dank
}

Ein Herausgeber blickt auf 45 Jahrgänge zurück

Mit der Vollendung des 45. Jahrgangs unserer Zeitschrift scheide ich aus dem Herausgeberteam von Communicatio Socialis aus. Ich war bei den Gründungsvorarbeiten 1967 und beim ersten Heft (JanuarMärz 1968) dabei - und bin es seither immer geblieben, nachdem der Erfinder der Idee, P. Dr. Franz Josef Eilers SVD, Karl Höller (damals Redakteur bei „Kirche und Leben“, Bistumsblatt Münster) und mich (damals Wissenschaftlicher Assistent am Institut für Publizistik der Universität Münster) zur Gründung und weiteren Zusammenarbeit eingeladen hatte. Heute stehe ich vor 1,32 Metern Communicatio Socialis in einem meiner original Billy-Regale und betrachte mit Respekt all die Ausgaben, die da zusammen gekommen sind. Ihr Entstehen war insofern Verdienst der Herausgeber, als sie für Kontinuität und Unermüdlichkeit gesorgt - und gelegentlich, gar nicht so selten, eigene Beiträge geliefert haben.

Niemals entstanden wären die 45 Jahrgänge ohne die zahlreichen Helfer, die, meistens ehrenamtlich, für die Beschaffung und Einrichtung der Manuskripte bis zur Druckfreigabe und schließlich, je länger je mehr, zur Erstellung der fertigen Druckvorlagen gesorgt haben und sorgen. Allein im redaktionellen Bereich waren es 17, davon acht Redakteurinnen und Redakteure im engeren Sinne. Ihnen allen möchte ich bei meinem Abschied danken, denn ohne sie wäre den drei Gründungsherausgebern, nachdem sie die Zeitschrift in den ersten drei Jahren auch als Redakteure allein besorgt hatten, bald die Luft ausgegangen.

Communicatio Socialis wurde, soweit es um Inhalte und formale Gestaltungsvorgaben geht, von drei Instanzen geprägt: dem Herausgeber-Team, der Redaktion und (von 1989 bis 2002) einem Redaktionsbeirat. (Für die Herstellung im technischen Sinne, den Vertrieb und die Werbung waren wechselnde Verlage zuständig. 1968 bis 1970 der Verlag Lechte in Emsdetten, 1971 bis 1993 Schöningh in Paderborn, 1994 bis 2005 der Matthias-Grünewald-Verlag Mainz und schließlich seit 2006 der Matthias-Grünewald-Verlag im Schwaben-Verlag Ostfildern.) Die Funktionen der drei inhaltsprägenden Instanzen waren mehr oder weniger deutlich differenziert, in der Aufgabenerfüllung jedoch zeitweise deckungsgleich, später teilweise überlappend. 
Das Herausgeber-Team umfasste seit Bestehen jeweils drei oder vier Personen. In seiner ersten Gestalt (Eilers/Höller/Schmolke, 1968-1988; 1971 bis zu dessen Tod 1983 erweitert um den niederländischen Verleger Kees Verhaak aus Nijmegen) bestand in ihm eine Art Hierarchie, die zwar niemals formalisiert wurde, jedoch durch die funktionale Autorität des Vaters der Idee, FJE, gegeben war. Ebenso formlos, bedingt durch die Notwendigkeiten (Eilers in Rom, Eilers in Genf etc.), entwickelte sich später die Rolle des geschäftsführenden Herausgebers. Karl Höller übernahm sie. Nach der Societas Verbi Divini (Steyler Missionare) konnte 1972 das Internationale Missionswerk Missio als Sponsor gewonnen werden. Mission und interkulturelle Kommunikation waren die ideelle Verbindung.

Ab 1989 kann man vom Herausgeberteam in seiner zweiten Gestalt sprechen. Von dieser Zeit an konnte Communicatio Socialis für die finanzielle Absicherung die vermittelnde Unterstützung der Medien Dienstleistungs GmbH in Anspruch nehmen, die - als Auswirkung der Würzburger Synode - 1975 von der Deutschen Bischofskonferenz (DBK) gegründet worden war. Seither ergänzt eine Vertrauensperson der Zentralstelle Medien der DBK das Herausgeberteam: von 1989 bis 1993 Peter Düsterfeld, 1993 bis 2002 Reinhold Jacobi, 2003 und 2004 Matthias Kopp und 2005 bis 2012 Ute Stenert - die beiden letzten schon für die neue DBK-Struktur „Bereich Kirche und Gesellschaft“.

\section{Strukturveränderung in der Herausgeberschaft}

Inzwischen hatte jedoch - mit der neuen Förderung - die größte Strukturänderung in der Herausgeberschaft stattgefunden. Franz Josef Eilers wurde 1988 auf Dauer von seinem Orden als Professor an das Divine Word Seminary in Tagaytay auf den Philippinen entsandt und übernahm zugleich in Manila die Leitung des Office of Social Communication der Asiatischen Bischofskonferenz. Karl Höller wurde Verleger in Aachen. Internationale Tätigkeit und wachsende berufliche Beanspruchung ließen 2002 beiden ein Aussscheiden aus dem Herausgebergremium ratsam erscheinen. Die nun folgende Ergänzung zum Triumvirat hing, was die Personen angeht, mit einer Veränderung der Grundstrukturen der Zeitschrift zusammen: Die Societas Verbi Divini, Eigentümerin der Titelrechte der Gründung ihres Mitglieds Eilers, verlieh 2003 diese Rechte an die Katholische Universität Eichstätt-Ingolstadt, und deren Präsident benannte den Eichstätter Professor Dr. Walter Hömberg, Inhaber des Lehrstuhls Journalistik I, zum Herausgeber seines Vertrauens. 
Matthias Kopp vom Bereich Kirche und Gesellschaft, der seit 1998 in der Communicatio Socialis-Redaktion mitgearbeitet hatte, wurde mit Heft 2/2003 Mitherausgeber. Schmolke blieb als Traditionsträger, und in dieser Zusammensetzung (Hömberg/Kopp/Schmolke) arbeitete das Herausgeberteam weiter, geschäftsführend geleitet von Hömberg. An die Stelle von Kopp trat Anfang 2005 Ute Stenert. Walter Hömberg legte mit Ende 2010 seine Herausgeber-Funktion nieder. Seinen Platz nimmt seither der Eichstätter Journalistik-Professor Klaus-Dieter Altmeppen ein, auch als geschäftsführender Herausgeber. Alexander Filipović ist 2012 Mitherausgeber geworden, gewissermaßen im Vorgriff auf meinen Rücktritt. Als Mitarbeiter des Instituts für Christliche Sozialwissenschaften an der Universität Münster hält er, wie der gebürtige Münsteraner Altmeppen, die Erinnerungen an den Gründungsort der Zeitschrift aufrecht.

\section{Die Rolle der Herausgeber}

Obwohl mein Abschiedsgruß weder eine Sammelbiografie der dramatis personae noch der Anfang einer Geschichte dieser Zeitschrift sein soll, habe ich bei den Herausgebern auch ein wenig den Hintergrund ihrer Rekrutierung und ihrer Berufe eingebracht. Das wird im Folgenden bei den Redaktionsmitarbeitern nicht so sein; die Darstellung soll, allein wegen der beachtlichen Zahl der Personen, auf die wichtigsten Daten beschränkt bleiben, also ins Chronistische übergehen. Der Grund liegt im einsichtigen Unterschied: Ohne Redakteure hätte die Zeitschrift nicht so werden und sein können, wie sie realiter geworden ist. Ohne Herausgeber aber hätte sie überhaupt nicht sein können.

Über den Herausgeber als publizistische Rolle ist noch nicht all$\mathrm{zu}$ viel geforscht worden, und hier und jetzt verweigere ich mich der Versuchung, gründlich zu klären, was das nun eigentlich ist: der Herausgeber eines Periodikums. Zwar gibt es im alten „Handbuch der Zeitungswissenschaft" (in der 6. Lieferung, 1942) ein eigenes Stichwort „Herausgeber“, im „dtv-Wörterbuch“ zur Publizistik jedoch nicht, auch keine Register-Erwähnungen, im „Fischer Lexikon Publizistik Massenkommunikation“ (Auflagen 1989 bzw. 1984) auch nicht. Das „Fischer Lexikon“ von 1971 sagt im Artikel „Journalist“, dass der „Chefredakteur für die Haltung der Zeitung oder Zeitschrift verantwortlich“ sei, „sofern der Herausgeber diese festgelegt hat". Das erklärt für unseren Zweck schon viel. Jörg Requate, in seinem "Journalismus als Beruf“ (1995), hilft weiter, indem er an die Entwicklungen im 19. Jahrhundert und, an Dieter Baumert anknüpfend, die Unterscheidung zwischen „Herausgeber- und Verlegerzeitungen“ 
vorschlägt. So gesehen war Communicatio Socialis stets ein Herausgeber-, aber oft auch ein Redakteursblatt, besonders in jenen Zeiten, als die Herausgeber in Personalunion Redaktionsmitglieder waren: 1968-1988 Eilers/Höller/Schmolke und 2008-2010 Hömberg.

Damit kehren wir aus dem Exkurs in den Abschnitt „HerausgeberTeam" zurück. Je mehr sich aufgrund der umfangreicher werdenden Aufgabenstellung eine funktionale Selbstständigkeit des Redakteurs (bzw. der Redaktion) herausschälte, desto deutlicher wird auf die Existenz von Richtlinien und auf Richtlinienkompetenz der Herausgeber hingewiesen, so z.B. im Verlagsvertrag mit Grünewald vom 27.2./11.3.2003 und in der Herausgebervereinbarung mit der Universität Eichstätt vom 6.2./25.2.2003. Nicht alle Richtlinien sind verschriftlicht. Die (bisher!) für den Inhalt geltende Kernrichtlinie (Orientierung an "Communio et Progressio“) findet sich im Anhang der Herausgebervereinbarung. Einiges $\mathrm{zu}$ den formalen Abläufen steht in der Protokollnotiz zu Paragraph 6 des Verlagsvertrags, in welcher die Zusammenarbeit zwischen Herausgebern und Redaktion geregelt ist. Aus Ziffer 4 kann man, ohne groß interpretieren $\mathrm{zu}$ müssen, herauslesen, dass das Herausgeber-Team im Hinblick auf den Umgang mit Inhalten ein Kollegialorgan ist, das z. B. bei der Begutachtung „umfangreicher bzw. erkennbar kontroverser Texte“ mit Mehrheit entscheiden darf und notfalls muss.

Die Mitarbeiter im redaktionellen Bereich (kurz Redaktion) lassen sich gliedern in „Personal-Unionisten“, Redakteure im engeren Sinne, redaktionelle Zuarbeiter in einem locker geregelten Verhältnis zur Redaktion und schließlich Redaktionsteams.

Die Personalunionen sind im Herausgeber-Abschnitt bereits genannt worden. Als erster Redakteur stieß Anfang 1971, also zum 4. Jahrgang, Josef Hosse zur Zeitschrift. 1914 in Dortmund geboren arbeitete er beim Verlag Schöningh und zuletzt beim „Ruhrwort“. Am 30. November 1982 starb er, nachdem er Heft 4/1982 am 12. Oktober noch fertig umbrochen hatte. Die redaktionelle Verantwortung fiel danach wieder (bis 1988) an die drei Erstherausgeber zurück, ungeachtet der Tatsache, dass sich seit 1968 nach und nach redaktionelle Helfer eingestellt hatten, die aus Liebe zur Sache mitarbeiteten: 1968-1987 Elmar Bordfeld, die ersten Jahre als römischer Korrespondent (heute noch freier Journalist und KNA-Mitarbeiter), 1969-1982 Michael Bornefeld-Ettmann (gestorben 1989, zuletzt Dezernent für Öffentlichkeitsarbeit der Stadt Münster), 1976-1987 Giso Deussen (heute als Professor der Fachhochschule Bonn-Rhein-Sieg im Ruhestand), 1985-1987 Manfred Becker-Huberti (seit 2007 Honorarprofessor der Philosophisch-Theologischen Hochschule Vallendar). 


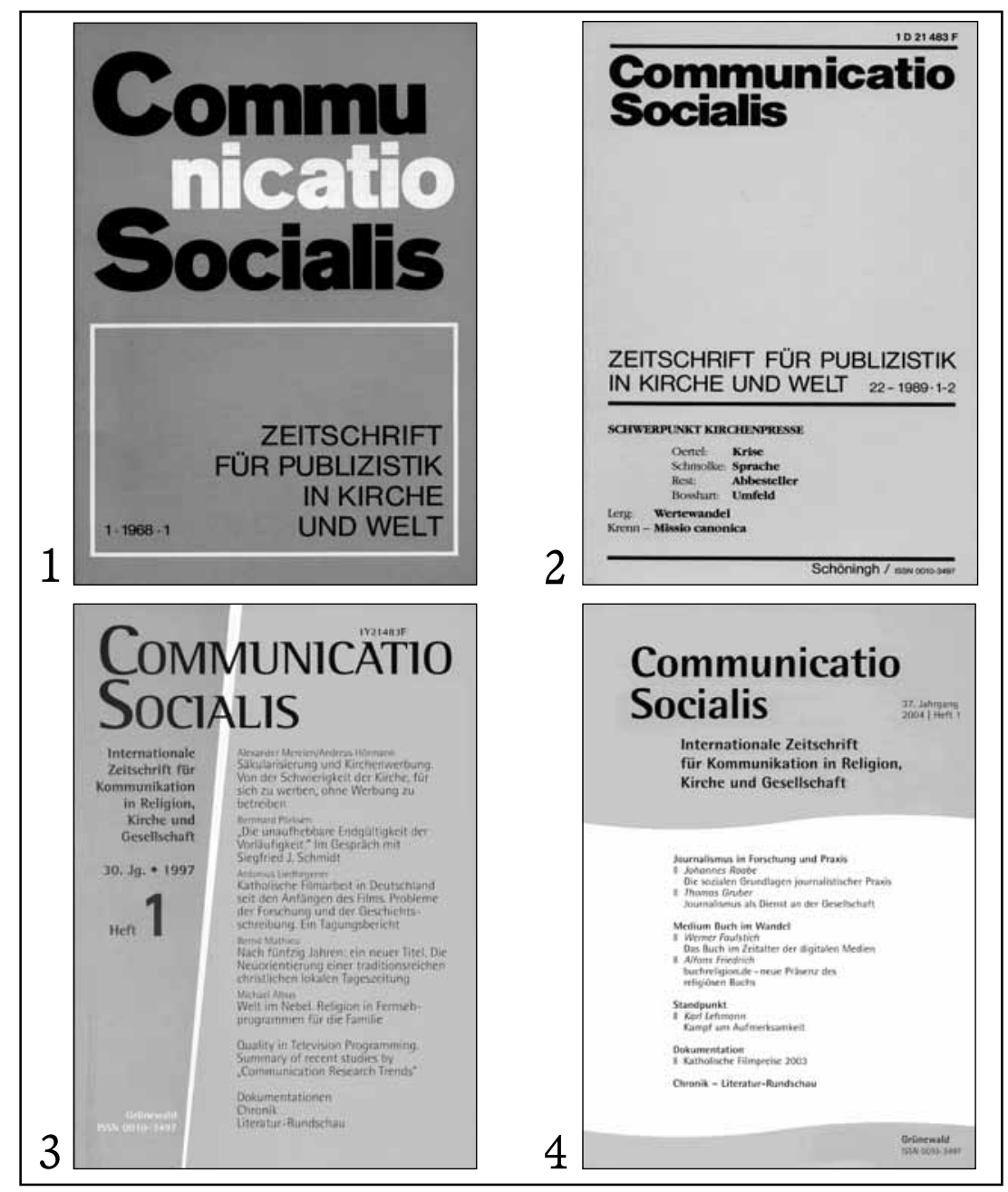

Communicatio Socialis wandelt sich: erste Ausgabe 1968 (1), neue Optik ab 1989 (2) und 1997 (3) und Titellayout seit 2004 (4).

Für 1983 bis 1986 kamen Jutta Bergmoser (heute Lektorin im Verlag Bergmoser und Höller, Aachen) und für 1986/87 Bernhard Meuser (heute St. Ulrich-Verlag, Augsburg) hinzu. Nach dem unfreiwilligen Sabbatjahr 1988, in dem nur das Registerheft für 1978-1987 erschien, übernahm die Salzburger Assistentin Gertraud Lankes die Redaktion, wurde aber schon 1990 von den Einspringern Rolf Pitsch und Hermann-Josef Große Kracht abgelöst, die der Redaktion auch erhalten blieben, als 1991 Helmuth Rolfes, Professor für systematische Theologie an der Universität Kassel, die Leitung der Redaktion übernahm. Er, der inzwischen emeritiert ist, war ein wesentli- 
cher Mitgestalter der Zeitschrift und blieb bis 2002, während Pitsch (heute beim Bonifatius-Verlag Paderborn) und Große Kracht (heute Akademischer Rat an der TU Darmstadt) sich 1996 bzw. 1999 anderen Aufgaben zuwandten. In der Rolfes-Zeit ergänzten Susanne Kampmann/Haferkamp (1997-2001; heute Wissenschaftliche Mitarbeiterin für Öffentlichkeitsarbeit an der Theologischen Fakultät der Universität Münster), Matthias Kopp (1998-2002; heute Pressesprecher der Deutschen Bischofskonferenz) sowie Ludger Verst (2002) die Redaktion.

Ein Sonderfall ist Ferdinand Oertel ${ }^{1}$, der ebenfalls 1991 fester Mitarbeiter der Rolfes-Redaktion wurde und der Zeitschrift bis zum Ende des Jahres 2012 erhalten blieb, zeitweilig als einspringender AlleinRedakteur (2003-2005). Er leitete die Redaktion, seit 2006 unterstützt von Renate Hackel-de Latour (Universität Eichstätt), bis 2007 und blieb der Redaktion auch für besondere Aufgaben verbunden, als Herausgeber Walter Hömberg für die Zeit von 2008 bis 2010 die Position des Chefredakteurs übernahm. Ebenfalls 2008 kam Christian Klenk (Universität Eichstätt) hinzu, und seit 2011 verstärkt die Eichstätter Absolventin Annika Franzetti das Team.

Von 1989 bis 2002 gab es außerdem einen Redaktionsbeirat. Er bestand aus den Professoren Louis Bosshart (Universität Freiburg/ Schweiz), Giso Deussen (Fachhochule Bonn-Rhein-Sieg), Joan Hemels (Universiteit van Amsterdam), Walter Hömberg (Katholische Universität Eichstätt), Michael Krzeminski (FH Bonn-Rhein-Sieg) und Rolf Zerfaß (Universität Würzburg). Man traf sich ein oder zwei Mal pro Jahr und verstärkte teils die herausgeberische, teils die redaktionelle Arbeit. So ist dann auch aus diesem Kreis der Herausgeber der Jahre 2003 bis 2010, Walter Hömberg, hervorgegangen.

Wenn ich die Herausgeber ausnehme, waren es, wenn ich niemanden übersehen habe, 17 Personen, die im Lauf der 45 Jahre in verschieden enger Bindung verschieden lange und verschieden intensiv die redaktionellen Arbeiten für Communicatio Socialis geleistet oder $\mathrm{zu}$ ihnen beigetragen haben, gemeinsam mit den Herausgebern, die immer wieder als Redakteure fungierten. Ohne diese 17 hätten die $\mathrm{He}-$ rausgeber, von den ersten drei Jahrgängen abgesehen, die Zeitschrift nicht machen können. Mit allen 17 habe ich in den verschiedenen $\mathrm{Ab}$ schnitten der 45 Jahre zusammengearbeitet. Ich danke ihnen und sage Adieu, was mein Duden (Ausgabe 1930) mit Gott befohlen übersetzt.

1 Oertels Rolle in der katholischen Publizistik im Nachkriegsdeutschland lässt sich nicht in einer Klammer-Notiz unterbringen. Man lese seine Erinnerungen: Der Kirchenzeitungsmann. Berlin ${ }^{2} 2012$. 